\title{
Comparison of particle in cell and fluid models in plasma display panels simulation
}

\author{
W. Benstâali ${ }^{1,2}$ and A. Belasri ${ }^{1}$ \\ ${ }^{1}$ Laboratoire de physique des plasmas, matériaux conducteurs et leurs applications LPPMCA, USTO'MB \\ University B.P 1505 EL M’NOUAR, Oran, Algeria \\ ${ }^{2}$ Faculté des Sciences de l'ingénieur, Ibn Badiss University, Mostaganem, Algeria
}

\begin{abstract}
In this paper, a particle in cell model (PIC) was developed. We compare the major characteristics of a single discharge obtained from our PIC model and the fluid model. Fluid model uses different approximations as Local field (LFA). The particle in cell (PIC) model, although its costs in time, still an efficient tool to understand a PDP discharge. Even there is some differences as the delay time and the ionization, the discharge still be correctly described as the energy balance.
\end{abstract}

\section{Introduction}

Despite the efforts of researchers in computer performance over the last decades, integrating all aspects of a system of plasma in a detailed model is not possible. Instead, simpler models are commonly used with appropriate assumptions. Well-organized simulation methods can provide physical information often difficult to obtain from experiments. Simulation results can also be used to identify research guidelines, find optimum operating conditions or propose novel designs for performance improvements. With this success, the numerical modeling of plasmas has become a valuable tool to understand and optimize plasma physics, especially plasma display panels (PDPs).

Plasma display panels (PDPs) are actually one of the principal technologies in flat display panels because of their large size (a 70" PDP is realised actually), their wide viewing angle $\left(\sim 180^{\circ}\right)$, their high colour resolution, and their flat body. Nevertheless, it still needs many improvements like the luminous efficiency which is only 1-2 $\mathrm{lm} / \mathrm{w}$. Although many experiences were realized to understand PDPs mechanism [1-3] and to give the ways to improve their performances, Modelling is an important tool to provide progress to this research effort. Several models were developed in order to study discharges in plasma display panels, their kinetic and the discharge efficiency [4-9]. Many numerical models have been also developed to study the different methods to optimize plasma display panel characteristics, particularly the luminous efficiency [10-14]. One way to increase the luminous efficiency is to improve the part of the input energy deposited by electrons into xenon excitation. This can augment U.V production, and then the visible light production. Several papers have study this way using fluid model [15-18]. This model use different approximations like local field approximation (LFA). The aim of this work is to compare our one dimensional particle in cell model with Mont Carlo Collisions model (PIC'MCC) [19-20] to the 1D fluid model used by Meunier et al [21]. The two models are presented in Section 2. In Section 3, are presented the characteristics of a single discharge described by both PIC and fluid model. The energy balance is then discussed.

\section{Physical model}

\subsection{PIC model}

The particle in cell (PIC) model consists of tracking macro- particles individually according to fundamental laws:

The charged particles trajectories are obtained by the Newton-Lorentz equations:

$$
\begin{aligned}
& m \cdot \frac{d \vec{V}}{d t}=\overrightarrow{\mathrm{F}} \\
& \frac{d X}{d t}=\mathrm{V} .
\end{aligned}
$$

Where $\mathrm{m}$ is the particle mass, $\mathrm{V}$ the velocity, $\mathrm{X}$ the position, and $\mathrm{F}$ the applied force

The electric field is obtained form Poisson's equation:

$$
\nabla^{2} \Phi=-\frac{\rho}{\varepsilon_{0}}
$$

Where $\Phi$ is the electric potential, $\rho$ is the charged density, and $\varepsilon_{0}$ is the permittivity, and $E=-\nabla \Phi$.

In PIC simulations, field quantities are known at the grid points whereas particles are allowed to fill the continuum phase space within the simulation domain. A typical algorithm involves basic steps as seen in Figure. 1 [21-24]. 


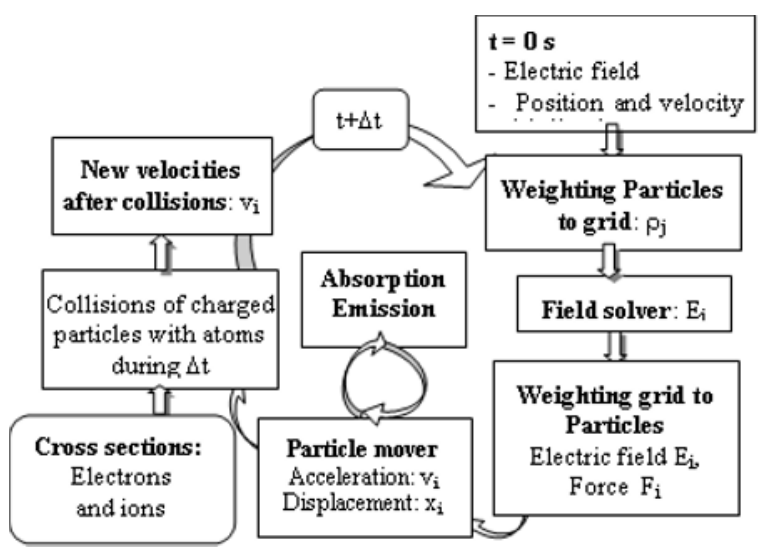

Fig. 1: Diagram of a PIC-MCC model.

The motion equations described above are discretized using the leapfrog scheme. In finite-difference form, we obtain the following equations:

$$
\begin{aligned}
& m \cdot \frac{V_{i}^{t+1 / 2}-V_{i}^{t-1 / 2}}{\Delta t}=F_{i}^{t}=q \cdot E_{i}^{t} \\
& \frac{X_{i}^{t+1}-X_{i}^{t}}{\Delta t}=V_{i}^{t+1 / 2} \\
& \Phi_{j+1}-2 \Phi_{j}+\Phi_{j-1}=-\frac{(\Delta x)^{2}}{\varepsilon_{0}} \cdot \rho_{j}
\end{aligned}
$$

where, $\mathrm{X}_{i}, \mathrm{~V}_{i}, \mathrm{~F}_{i}$, and $\mathrm{E}_{i}$ are respectively the position, the velocity, the force, and the electric field at the particle position. $\Phi_{j}$ is the potential at grid point $j, \Delta \mathrm{t}$ is the time step, and $\Delta \mathrm{x}$, is the distance between grid points.

The electric field at grid point $j$ is deduced from the potential:

$$
E_{j}=\frac{\Phi_{j-1}-\Phi_{j+1}}{2 . \Delta x}
$$

The electric field at each position is calculated as follows (Weighting grid to Particles):

$$
E_{i}=E\left(X_{i}\right)=\left(\frac{\mathrm{x}_{\mathrm{j}+1}-X_{i}}{\Delta x}\right) \cdot \mathrm{E}_{\mathrm{j}}+\left(\frac{\mathrm{X}_{\mathrm{i}}-x_{j}}{\Delta x}\right) \cdot \mathrm{E}_{\mathrm{j}+1}
$$

where $\mathrm{E}_{j}$ et $\mathrm{E}_{j+1}$ are electric fields at $j$ and $j+1$ respectively, $\mathrm{x}_{j}$ et $\mathrm{x}_{j+1}$ are the positions of adjacent cells, $\mathrm{X}_{i}$ is the particle position. $\Delta \mathrm{x}$ is the space between two neighbor cells, it is equal to the total gas space distance on the number of grids

The particle collisions are studied by the Monte Carlo collisions method [25-26]. The time step used in this work is taken as small as possible to make the collision probability per time step very small. Each time step we check only for a limited number of particles whether they collide. Number of macro-collisions per time step $\mathrm{Nc}=$ $\operatorname{int}(\mathrm{Np} v \max d t)+1$, can be chosen at random from the particle list allowing for each particle to be drawn more than once. Once the particles undergoing collisions have been selected, the type of collision for each particle is determined by choosing a random number. For this reason, we need to know the appropriate cross sections;
The ion and electron collision cross sections are given by Piscitelli et al. [27] and in Bolsig+[27] respectively.

\subsection{Fluid model}

The one-dimensional fluid model is based on the continuity and the simplified momentum-transfer equations for electrons and ions, coupled with Poisson's equation for the electric field [21]:

$$
\begin{gathered}
\frac{\partial \mathrm{n}_{\mathrm{e}}}{\partial t}+\nabla \cdot\left(n_{e} \bar{v}_{e}\right)=S_{e} \\
n_{e} \bar{v}_{e}=-n_{e} \mu_{e} E-D_{e} \nabla n_{e} \\
\frac{\partial \mathrm{n}_{\mathrm{p}}}{\partial t}+\nabla \cdot\left(n_{p} \bar{v}_{p}\right)=S_{p} \\
n_{p} \bar{v}_{p}=n_{p} \mu_{p} E-D_{p} \nabla n_{p} \\
\nabla \varepsilon_{r} \mathrm{E}=\frac{|\mathrm{e}|}{\varepsilon_{0}}\left(n_{p}-n_{e}\right) .
\end{gathered}
$$

Where $n_{e}$ is the electron density, $n_{p}$ the positive ion density. The index $p$ in the equations refers to xenon or neon ions. $\bar{v}_{e}$ and $\bar{v}_{p}$ represent the mean velocity for electrons and ions, respectively. $S_{e}(\mathrm{r}, t)$ and $S_{p}(\mathrm{r}, t)$ are the production rates for electrons and ions respectively. The mobilities $\mu_{e}$ and $\mu_{p}$ are function of the reduced electric field $E / p . D_{e}$ and $D_{p}$ are respectively the electrons and ions diffusion coefficients. The $\mathrm{D} / \mu$ are set to constant values. $\varepsilon_{\mathrm{r}}$ is the relative permittivity and $\mathrm{e}$ is the unit charge. Equations (9) and (11) are the electron and positive-ion continuity equations, Eq. (10) and (12) are the simplified momentum-transfer equation for electrons and ions, and Eq. (13) is Poisson's equation.

\section{Results}

In this section we compare results obtained from the $1 \mathrm{D}$ models described above for a single discharge pulse in a mixture of $10 \%$ xenon in neon. The gap length $\mathrm{d}$ is $100 \mu \mathrm{m}$, the pressure $\mathrm{p}$ is 560 Torr, and the equivalent capacity of dielectric layers is $230 \mathrm{pF} / \mathrm{cm} 2$. The gas temperature is supposed to be constant and equal to 300 ${ }^{\circ} \mathrm{K}$. The secondary emission coefficients of xenon and neon are 0.005 and 0.5 respectively. The scheme of the simulated cell is shown in Figure 2.

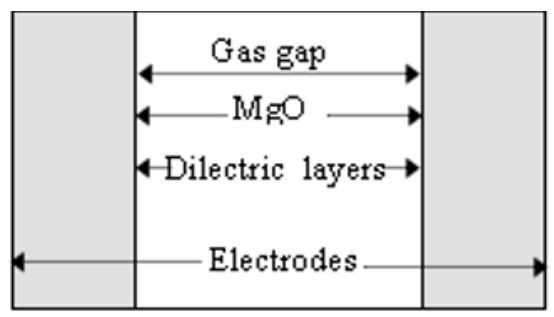

Fig.2: Schematic of the simulated PDP cell. 


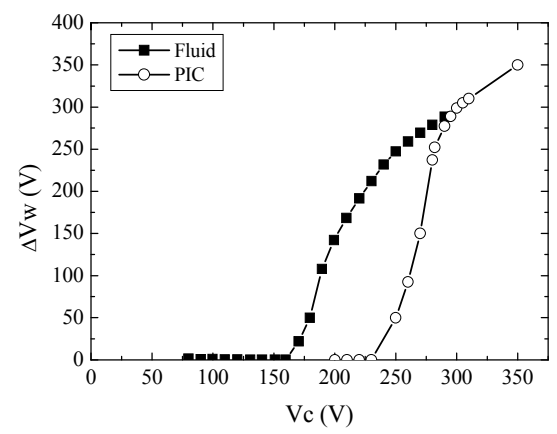

Fig.3: Voltage curve $\Delta \mathrm{Vw}=\mathrm{f}(\mathrm{Vc})$ for discharge cell in $\mathrm{Xe10}-\mathrm{Ne}$ mixture for PIC and fluid models

Figure 3 presents the voltage transfer curve obtained with PIC and fluid models [21]. It is observed that for PIC model, the minimum and maximum values, $\mathrm{V}_{\text {smin }}$ and $\mathrm{V}_{\text {smax }}$ of the sustaining voltage for bistable operations [21,29] are $151 \mathrm{~V}$ and $230 \mathrm{~V}$. For fluid model, $\mathrm{V}_{\text {smin }}$ is of $124 \mathrm{~V}$ and $\mathrm{V}_{\text {smax }}$ is of $160 \mathrm{~V}$. The margins are then, $79 \mathrm{~V}$ and $36 \mathrm{~V}$ for PIC and fluid models respectively. Consequently, if we use the sustaining voltage in the mid-margin, $\mathrm{V}_{\text {mid-margin, }}$ the applied voltage to the single discharge, which is twice $\mathrm{V}_{\text {mid-margin }}$, is $381 \mathrm{~V}$ for PIC model and $284 \mathrm{~V}$ for fluid model.

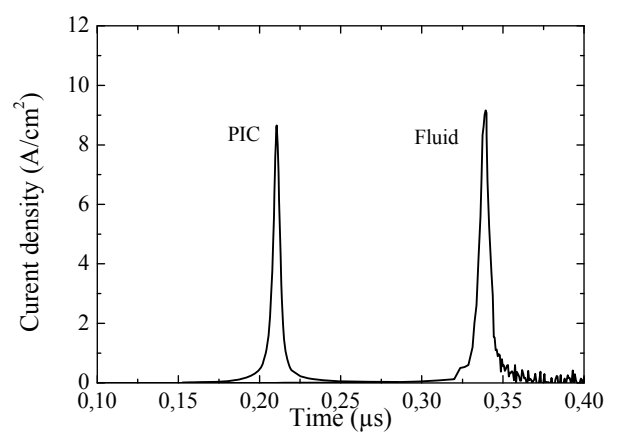

Fig.4: Temporal variation of current density for a discharge in a Xe10-Ne mixture.

As it is shown, the discharge can be initiated by fluid model at voltage less than by PIC code although the same electron cross sections are used. This can be explained by the fact that the ionization coefficient in Monte Carlo Collisions is less obtained from Boltzmann equation. Furthermore, the ionization coefficient is considered to be constant in the entire cell in fluid model when it isn't in PIC model which induces a less ionization in this model. The question is does it affect the discharge characteristics?

In Figure 4 is represented the current density of a single discharge for the conditions cited above. As the voltage is applied to the cell, the charges migrate toward dielectrics and the breakdown occurs at $200 \mathrm{~ns}$ for fluid model and at $320 \mathrm{~ns}$ for PIC model. The current density increases and reaches a peak of 9 and 8.5 for PIC and
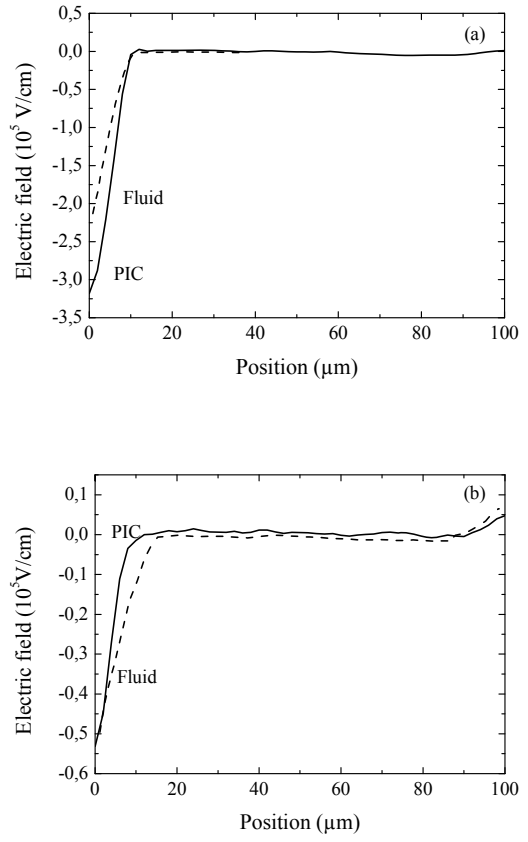

Fig.5: Spatial distribution of the electric field for PIC and fluid models: (a) at the maximum of current, (b) at the post-

discharge. The gap length is $100 \mathrm{pm}$, the gas pressure is 560 Torr $(300 \mathrm{~K})$; the capacitance of each dielectric is $460 \mathrm{pF} / \mathrm{cm}^{2}$.

fluid model respectively. Even if the delay time is more important for PIC model, the peak of current is almost in the order of that obtained from fluid model. This means that the difference between fluid and PIC model affects only the pre-breakdown and not the discharge. This can be verified subsequently. It is also, seen in Figure 5(a) that, at the peak of current, the electric field reaches a value of $310^{5}$ and $2.110^{5} \mathrm{~V} / \mathrm{cm}$ for PIC and fluid models respectively, when the cathode sheath length is in order of 8 and $10 \mu \mathrm{m}$ for PIC and fluid model respectively. After the extinction of discharge; post discharge, fluid model shows a cathode sheath larger than that of PIC model (see Figure 5 (b)). It is noted in Figure 6 (a)-(d) that the two models give the same allure of charges densities at the maximum of current and at the postdischarge. There are no electrons at the cathode sheath. At the peak of current, electron density is more important in PIC model; in order of $3.510^{14} \mathrm{~cm}^{-3}$ when it is $5.710^{13}$ $\mathrm{cm}^{-3}$ in fluid model. While the positive charge density in fluid model is larger than in PIC model $\left(10^{14}\right.$ and $610^{13}$ $\mathrm{cm}^{-3}$ respectively).

Figure 7 represents the diagram of energy balance obtained with the two models. It is noted that from the total energy deposited in the discharge, PIC model estimates that only $40 \%$ is used by electrons when Fluid model give approximately $42 \%$. It is shown in the two cases that the major part of this energy contributes to xenon excitation and ionization. However, the ionization is more important in fluid result (18\%) than in PIC result $(13.3 \%)$ as we have expected previously. It is also shown from PIC results that there is $2 \%$ of the total energy lost at walls. This is not considered by fluid model. 

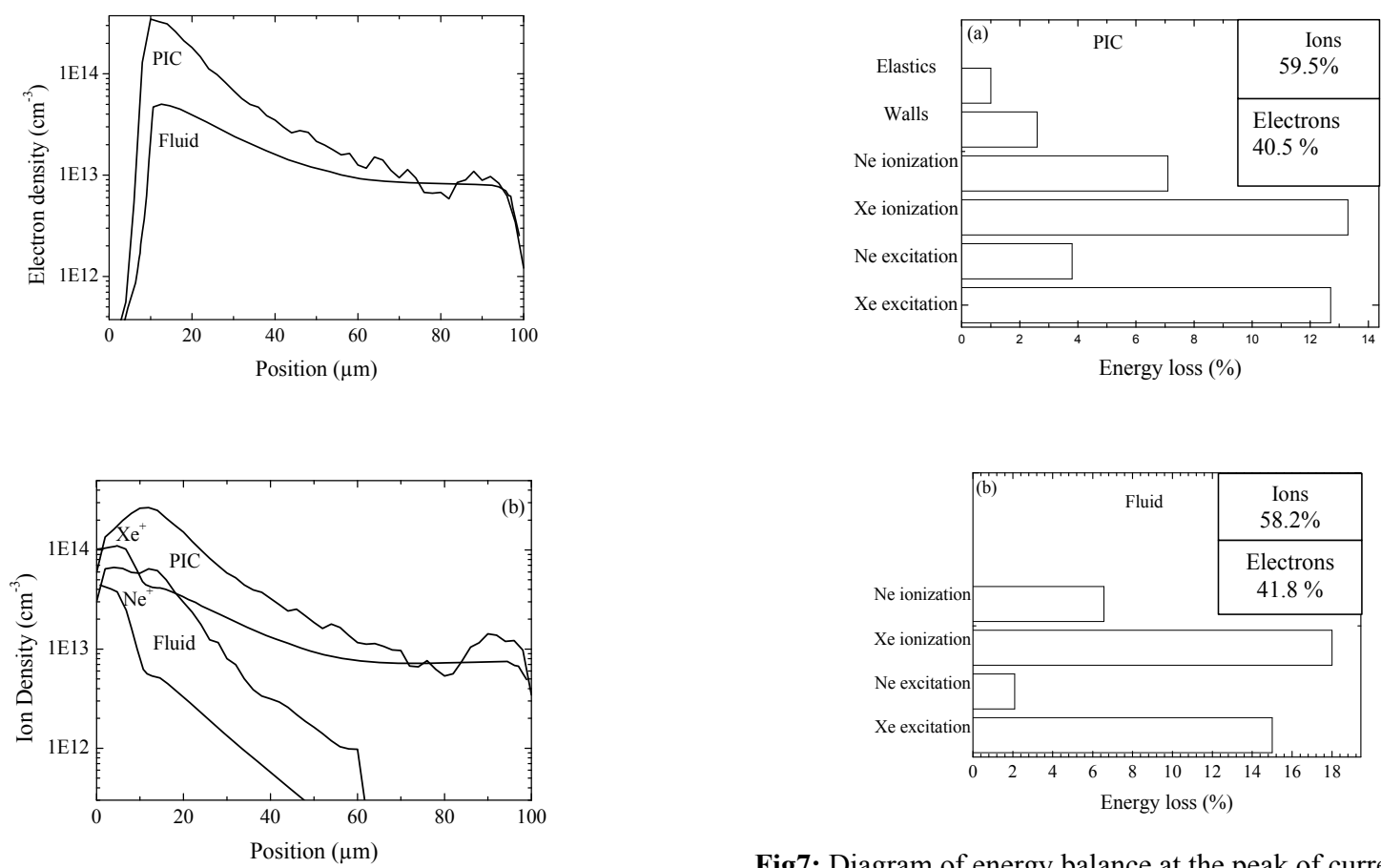

Fig7: Diagram of energy balance at the peak of current for PIC and fluid models
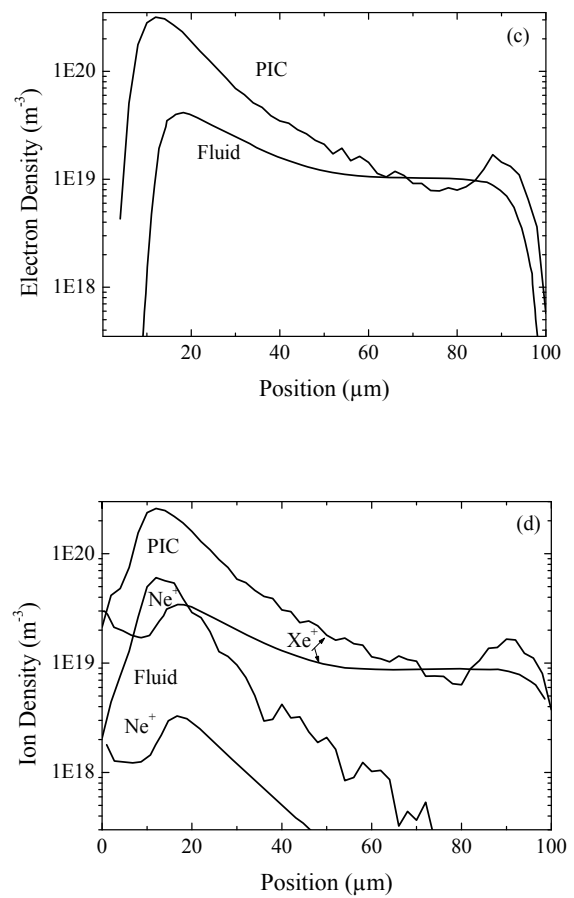

Fig.6: Spatial distribution of charged particles for PIC and fluid models: at the maximum of current; (a) electron density, (b) xenon and neon ions, and at post-discharge; (c) electron density, (d) xenon and neon ions.

Even if there is a difference between PIC and fluid model, the xenon excitation is affected only by $2.3 \%$ (12.7\% and $15 \%$ from PIC and Fluid models). The energy balance is well described by both fluid and PIC models.

The question was "Is the discharge energy distribution behaviour affected by these differences?" For this reason, a parametric study was investigated using our PIC model. A comparison with fluid model results obtained from bibliography is also done.

\section{Conclusion}

In this paper, we compare between particle in cell (PIC) and fluid methods. Although Fluid model uses Local field (LFA) which doesn't capture all the complexity of the non local electron transport, there is a good agreement between Fluid and PIC results. Ionization is more important in fluid model and the sustain voltage is higher in PIC model. This difference doesn't affect considerably discharge behavior. Energy balance is well described by both models. Only $2 \%$ of difference concerns xenon excitation.

The particle in cell (PIC) model, although its costs in time, still an efficient tool to understand a PDP discharge.

\section{Acknowledgments}

The authors would like to thank J.P.Boeuf and G.J.M Hagelaar for their help in the development of the model presented in this paper and for the fruitful discussions about energy loss mechanisms in plasmas.

\section{References}

[1] Th. Callegari, R. Ganter, and J.P. Boeuf, J. Appl. Phys, 88, 3905.(2000).

[2] Y. Ikeda, K. Susuki, H. Fukumoto, M. Shibata, M. Ishigaki, J.P. Verboceoeur, P.J Christenson, and C.K Birdsall, "Global breakdown in alternating current plasma display panel,” J.Appl.Phys, 89, 4231 (2001). 
[3] T. Sakurai, S. Matsuzawa, and Y. Kamo, J.Appl.Phys, 91, 4806 (2002).

[4] B.J. Shin, K.C. Choi, H.S. Tae,and S.S. Park, IEEE Trans. Plasma Sci, 33, 1426 (2005).

[5] M. Kurihara and T. Makabe, J.Appl. Phys, .89, 7756 (2001).

[6] J.P. Boeuf and. L.C. Pitchford, IEEE Tran.Plasma. Sci. Vol.24, 95 (1996).

[7] C. Punset, J. P. Boeuf and L. C. Pitchford, J. Appl. Phys. 83, 1884 (1998).

[8] S. Rauf and M. J. Kushner, J.Appl. Phys, 85,3460 (1999).

[9] Y. Ikeda , J.P. Verboncoeur, P.J. Christenson, and C.K. Birdsall, J.Appl.Phys, 86, 2431 (1999).

[10] S. Rauf and M. J. Kushner, J. Appl. Phys. 85, 3470 (1999).

[11] S.S. Yang, H.C. Kim, S.W Ko and J.K. Lee, IEEE Trans. Plasma Sci, 31, 596 (2003).

[12] G. Veronis and U.S. Inan, IEEE Trans. Plasma Sci, 33, 147 (2005).

[13] T.J. Kim, J.K. Kim, and K.W. Whang, IEEE Trans. Plasma Sci, 35, 1775 (2007).

[14] K.C. Choi, N.H. Shin, K.S. Lee, B.J. Shin, and S.E. Lee, IEEE Trans. Plasma Sci. 34, 385 (2006).

[15] G. J. M Hagelaar, M. H. Klein, R.J.M.M. Snijkers, and G. M. W. Kroesen, J.Appl.Phys. 89, 2033 (2001).

[16] W.J. Chung, B.J. Shin, T.J. Kim, H.S. Bae, J.H. Seo, and K.W. Whang, IEEE Trans. Plasma Sci, 31, 1038 (2003).

[17] D. Hayashi, G. Heusler, G. Hagelaar, and G. Kroesen, J.Appl.Phys. 95,1656 (2004).

[18] H.S. Bae, J.K. Kim, and K.W. Whang, IEEE Trans. Plasma Sci, 35, 467 (2007).

[19] W. Benstâali and A. Belasri, IEEE Trans. Plasma Sci, 39, 1460 (2011).

[20] W. Benstâali, Z.Harrache and A. Belasri, Pys.Scr, 85, 065502 (2012).

[21] J. Meunier, P. Belenguer and J. P. Boeuf, J. Appl. Phys, 78, 731 (1995).

[22] C. K. Birdsall and A. B. Langdon, "Plasma Physics via Computer Simulation," New York: McGraw-Hill, 1985.

[23] J. P. Verboncoeur, M. V. Alves, V. Vahedi, and C.K. Birdsall, "J. Comput. Phys, 104, 321 (1993).

[24] V. Vahedi and G. DiPeso, J. Comput. Phys, 131, 149 (1997).

[25] V. Vahedi and M. Surenda, Comput, Comput.Phys Commun, 87, 179 (1995).

[26] J.P. Verboncoeur, Plasma Phys. Control. Fusion, 47, A231 (2005).

[27] D. Piscitelli, 1.A.V. Phelps, J de Urquijo, E. Basurto, and L.C. Pitchford, Phys. Rev. E, 68, 046408-1 (2003).

[28] BOLSIG +2008 , www.laplace.univ.tlse.fr/ groupesde-recherche /GREPHE /projets-en-cours /Bolsig+ Résolution.

[29] S. Kawano and T. Misaka, J. Appl. Phys, 98, 053302 (2005). 